\title{
Mouvements de versants des retenues hydroélectriques Retour d'expérience et gestion du risque
}

\section{THOMAIIDIS}

Institut géologique

Albert-Lapparent

13, bd de l'Hautil 95092 Cergy-Pontoise

Cedex

thomaidis@caramail.com

\section{G. DEVEZE} J.-Y. DUBIE

Électricité de France,

Département TEGG,

Division Géologie

Géotechnique

905, av, du Camp-de-Menthe 13097 Aix-en-Provence Cedex 02 guilhem.deveze@edf.fr

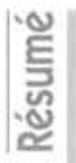

L'étude des versants surplombant les retenues revêt une importance capitale au regard de la sûreté de l'ouvrage et de la sécurité des populations. Les risques concernent principalement la submersion du barrage par une vague induite par un glissement en masse et l'endommagement des organes vitaux du barrage à la suite d'écroulements sur l'ouvrage,

La politique de gestion des mouvements de terrain à Électricité de France s'appuie bien sûr sur son expérience en termes de glissements déclarés (notamment ceux du Billan et du Chastel) mais aussi sur un inventaire préventif sur l'ensemble des retenues de ses 67 grands barrages. Cet inventaire s'intègre dans le cadre réglementaire des plans particuliers d'intervention (PPI). En cas de crise, le recueil d'un maximum d'informations, se révélant souvent par la suite précieuses, doit être privilégié. Cela nécessite à la fois une sensibilisation accrue de l'exploitant et une connaissance précise des zones à risque. L'évaluation géologique de l'aléa, en terme de vitesse, volume et facteurs déclenchants, permet parfois d'en limiter l'impact sur l'exploitation de l'aménagement hydroélectrique. Cette analyse préalable permet également une maitrise financière des travaux à engager.

Mots-clés : barrages, mouvements de versants, surveillance.

\section{Hydroelectric reservoirs landslides Case histories and risk management}

The stability study of reservoir slopes is of major importance for safety of both dam and downstream populations. Risks mainly concern creation of waves induced by sliding rocky masses which could submerge the dam. Damages on the dam resulting from cirect block falls are considering too.

Reservoirs landslides management at Electricité de France stands both on its specific experience of active slides and on a preventive inventory of these special hazards all around its 67 big dams reservoirs. This inventory is included in $\alpha$ Plan particulier d'intervention v regulation studies. During a slide crisis, acquiring maximum data is a priority. This requires both the sensibilization of the exploitant with the potential hazard and the accurate localization of unstable areas. The early evaluation of geological hazard (slide velocity, slide mass volume and causative factors) sometimes limits the negative impact of an unstable area on power station running. This analysis allows to optimize the cost of possible later investigations, monitoring system or reinforcing works.

Key words : dams, slope movements, monitoring. 


\section{Introduction - Problématique des glissements de terrains dans les retenues}

Les conséquences des mouvements de terrains sur les ouvrages hydrauliques sont plus ou moins importantes selon le stade de la vie de l'ouvrage impacté :

1) durant la construction, des déstabilisations de volumes à l'équilibre limite peuvent apparaître lors de l'ouverture de carrière, d'emprunt, de rétablissement des communications ou des fouilles méme du barrage. L'exposition au risque concerne alors le chantier, plus rarement les populations :

2) durant la mise en eau, les modifications des écoulements hydrauliques au sein du versant peuvent conduire à l'apparition ou à la réactivation de mouvements d'ensemble du versant. Cette phase, de loin la plus critique, peut menacer à la fois la pérennité de l'ouvrage mais également les populations à l'aval : 3) durant la période d'exploitation de l'aménagement, les mouvements de versant peuvent avoir trois effets sur la disponibilité et la sûreté de l'aménagement : - impact direct sur le barrage, pouvant endommager des organes vitaux de l'ouvrage (vannes, bâtiment de commande...),

- création d'un " barrage » naturel dans la retenue, par partition le mouvement de terrain vient boucher la retenue jusqu'à une cote supérieure à la cote normale d'exploitation) ou par obstruction (la hauteur de bouchon est inférieure à la cote normale, et des problèmes peuvent apparaître lors de vidanges),

- création de vagues pouvant submerger le barrage. La catastrophe de Longarone en Italie illustre tragiquement ce dernier (Chowdhury, 1978). Le 9 octobre 1963, un glissement de terrain d'un volume supérieur à $300 \mathrm{hm}^{3}$ descendit du mont Toc à une vitesse supérieure à $50 \mathrm{~km} / \mathrm{h}$, éjectant $50 \mathrm{hm}^{3}$ d'eau de la retenue du barrage du Vaïont. Le barrage voûte ne fut pas endommagé, mais la vague générée par le glissement submergea l'ouvrage de plus de $100 \mathrm{~m}$ et déferla dans la vallée, faisant près de 2000 victimes.

Électricité de France possède une expérience unique dans la gestion de mouvements de versants dans des sites hydroélectriques parfois sensibles. La gestion de ces mouvements de versant est soumise à l'approbation du Comité technique permanent des grands barrages (CTPB).

Cette expérience concerne :

- la gestion de mouvements de terrains déclarés :

- la gestion préventive de glissements potentiels susceptibles de mettre à mal la sûreté des ouvrages. Cette gestion préventive s'appuie sur le cadre réglementaire des plans particuliers d'intervention (PPI).

\section{2}

\section{La gestion des mouvements de versant declarés}

Compte tenu de la sensibilité des ouvrages, l'ana166 lyse rapide d'un mouvement de versant lors d'une phase paroxysmale est primordiale pour la sûreté de l'ouvrage.

Nous illustrons ci-dessous, pour trois cas distincts, la démarche retenue par EDF lors de l'apparition de mouvement de versants :

- le glissement du Chastel, pour lequel le risque de création d'une vague pouvant submerger le barrage est réduit et le risque partition a été jugé maîtrisable par l'exploitant; un simple dispositif de suivi a été mis en place;

- le glissement du Billan, où le glissement, qui représentait un risque pour la süreté de la retenue (risque de création de vague) a été conforté par drainage :

- les instabilités de la carrière du barrage de Grand'Maison où les zones instables qui menaçaient les travaux d'extraction n'ont pu être traitées. Elles ont entrainé une extension de la carrière dans une zone non exposée au risque et n'ont été auscultées que sur quelques années.

\section{1}

\section{Le glissement du Chastel (retenue du barrage de Puylaurent, Lozère)}

Le barrage de Puylaurent, sur le Chassezac (Lozère), est un barrage voûte à buts multiples EDF-SELO, d'une hauteur de $80 \mathrm{~m}$. Il se développe au sein des formations métamorphiques de l'ensemble cévenol. Au lieu-dit les Cotes-del-Chastel, à $2500 \mathrm{~m}$ à l'amont rive gauche du barrage, le versant de la retenue est composé de gneiss leptynitiques massifs et de micaschistes, peu altérés mais fortement fracturés.

En mai 1996, lors de la mise en eau du barrage, un glissement fut signalé à cet endroit, affectant le versant sur une hauteur de 100 m environ. Un dispositif d'auscultation provisoire, résumé dans le tableau I, fut mis en place et la décision fut prise de baisser le plan d'eau à une vitesse inférieure à $1 \mathrm{~m} / \mathrm{j}$.

ThaLEAU Détail du dispositif sommaire d'auscultation mis en place sur le glissement du Chastel.

Chastel slide : monitoring system.

\begin{tabular}{l|l}
\hline \multirow{2}{*}{ Déformations } & 3 profils de repères de déformation \\
\cline { 2 - 2 } & jalonnements \\
\cline { 2 - 2 } & 2 inclinomètres \\
\hline Topographie & 2 points \\
\hline Pièzométrie & 1 piézomètre \\
\hline
\end{tabular}

L'analyse des données du dispositif sommaire d'auscultation et l'interprétation de trois sondages carottés réalisés pour l'occasion permirent de déterminer les caractéristiques de ce glissement, d'un volume total compris entre 220000 et $250000 \mathrm{~m}^{3}$.

La figure 1 (Bozonnet, 1996) illustre les caractéristiques de ce glissement. La surface de glissement est définie par un plan de faille penté de $55^{\circ}$ vers la retenue, relayé par la foliation (pendage variant de 20 à $55^{\circ}$ vers la retenue). Le glissement affecte toute la hauteur du versant sur une hauteur de $120 \mathrm{~m}$ entre les 


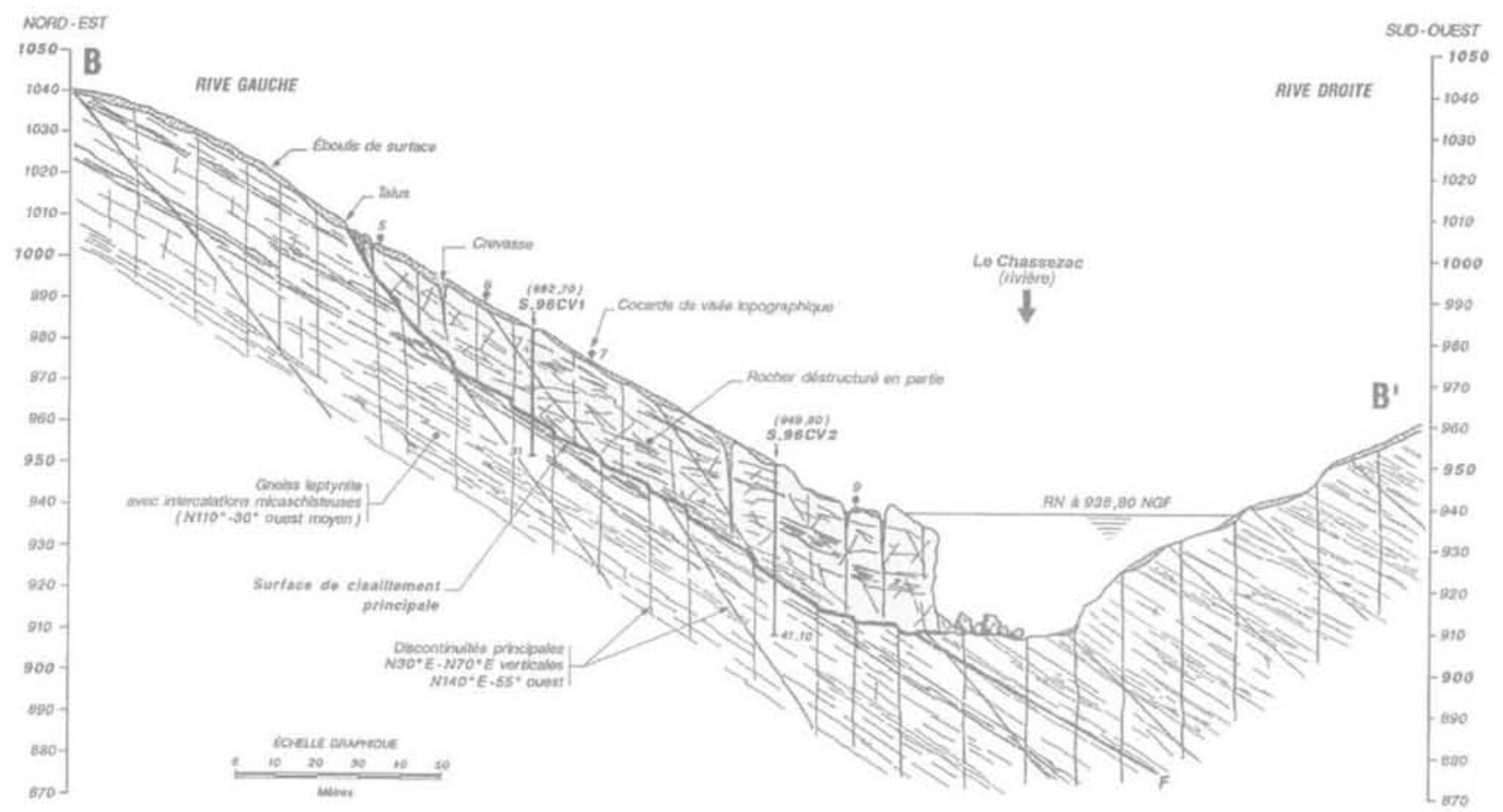

FiG.1 Coupe géologique du glissement du Chastel.

Geological section through Chastel slide.

cotes 907 et 1030 NGF. La base du glissement est donc sous le niveau de la retenue normale.

Le déplacement du glissement initial a été estimé entre 3 et $5 \mathrm{~m}$. Il a généré en tête une niche d'arrachement avec un rejet de 1 à $2,50 \mathrm{~m}$ et un fort affaissement de la zone située au-dessus de cette niche d'arrachement.

Par la suite, les vitesses se sont stabilisées autour de $0,5 \mathrm{~cm} / \mathrm{jour}$ pendant l'été 1996. Le glissement évolua ensuite vers différentes loupes, déstructurant la masse glissée jusqu'à n'être plus qu'un éboulis plus ou moins instable.

Le risque vague fut écarté pour plusieurs raisons : 1) le pied du glissement est sous la cote de la retenue normale :

2) la vallée resserrée assurera rapidement une butée de pied à un hypothétique glissement brutal :

3) la morphologie du glissement paraît inadaptée à un glissement présentant des vitesses élevées ;

4) l'évolution progressive du glissement semble écarter tout risque de rupture brutale.

Le risque obstruction/partition demeure, mais le profil de la vallée et les caractéristiques du glissement permettront une maitrise du plan d'eau en cas de crise.

\section{2}

\section{Le glissement du Billan (retenue du barrage de Grand'Maison, Isère)}

La retenue de Grand'Maison est le bassin supérieur de la station de transfert d'énergie par pompage de l'aménagement du même nom. Le barrage de Grand'Maison est un barrage en terre à noyau argileux, d'une hauteur de $140 \mathrm{~m}$, barrant l'Eau d'Olle et créant une retenue de $137 \mathrm{hm}^{3}$.
Le versant rive droite de la retenue est constitué en partie supérieure par les formations cristallines de Belledonne et en partie inférieure par des calcaires liasiques. Ces deux formations sont fortement affectées par une schistosité subverticale. Le contact cristallin/Lias est conforme à cette schistosité.

Des phénomènes importants de fauchage affectent une partie du versant, à la faveur de cette schistosité parallèle à la retenue au sein d'un versant très pentu (Fig. 2). L'épaisseur de la zone fauchée atteint la centaine de mètres, sur un dénivelé de $500 \mathrm{~m}$.

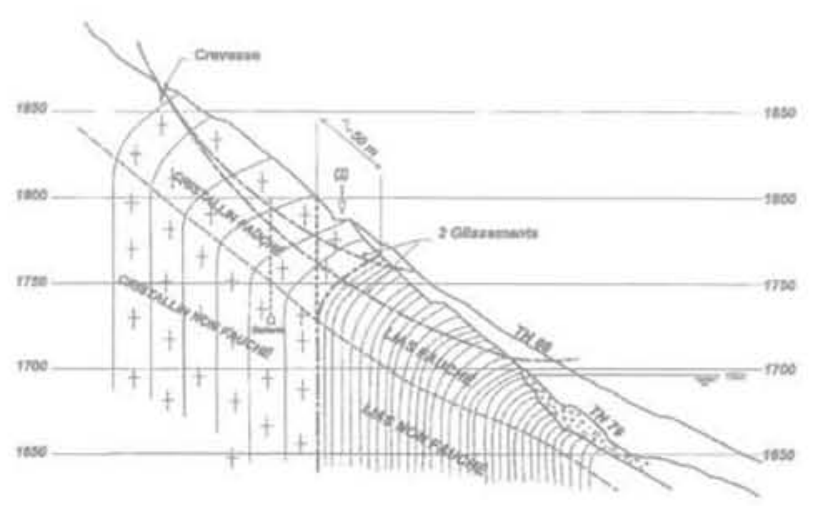

FIG.2 Glissement du Billan : coupe interprétative du versant, montrant le phénomène de fauchage.

Billan slide : interpreted section showing downbending phenomena.

Lors de la mise en eau du barrage, alors que la retenue atteignait la cote 1674 NGF (cote de projet : 1695 NGF), des fissures et un affaissement apparurent sur la route en rive droite, $3 \mathrm{~km}$ à l'amont du barrage. 
Des repères provisoires de déformations enregistrèrent le lendemain une augmentation de l'ouverture des fissures de près de $1 \mathrm{~cm}$. Le même jour, une crevasse de $250 \mathrm{~m}$ de longueur et d'environ $50 \mathrm{~cm}$ de rejet vertical fut découverte dans le versant à la cote $1850 \mathrm{~m} \mathrm{NGF}$ (Fig. 3).

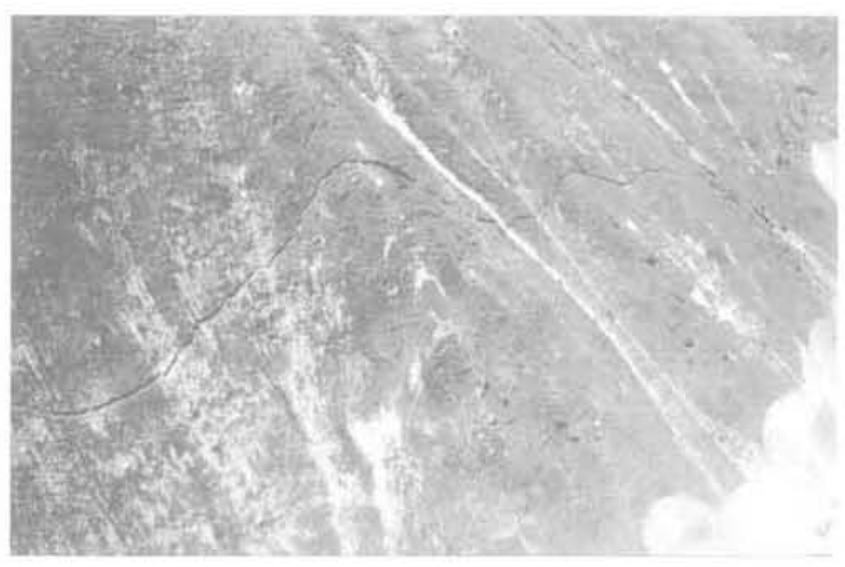

FG, 3 Crevasse de tête du glissement du Billan. Billan slide : Uphill tension crack.

Un dispositif d'auscultation sommaire permettant un suivi en continu fut mis en place. Il est résumé dans le tableau II.

Le plan d'eau fut baissé jusqu'à la cote 1660 à la vitesse de $1 \mathrm{~m} / \mathrm{j}$.

Une analyse rapide des faits conduisit à attribuer la réactivation de ce glissement à une remontée exceptionnelle de la piézométrie au sein du versant suite à un

TABLEAU Dispositif de crise et dispositif de drainage du glissement du Billan.

Billan slide : crisis monitoring system and drainage facilities.

\begin{tabular}{|c|c|}
\hline Sismique réfraction. & 16 dispositifs \\
\hline \multirow[t]{2}{*}{ Sondages } & 14 sondages destructifs \\
\hline & 10 sondages carottés (2 $000 \mathrm{~m})$ \\
\hline Piézométrie & 10 piézomètres \\
\hline Hydrologie de surface & Suivi en débit de 9 sources \\
\hline Déformation & 8 inclinomètres \\
\hline \multirow[t]{3}{*}{ Déplacements. } & $\begin{array}{l}51 \text { points de nivellement } \\
\text { sur la route }\end{array}$ \\
\hline & Géodésie (45 points sur 3 profils) \\
\hline & $\begin{array}{l}\text { Aéro-triangulation } \\
\text { par photogrammétrie analytique } \\
(350 \text { cocardes réparties } \\
\text { sur } 1750 \text { ha) }\end{array}$ \\
\hline Galerie & $\begin{array}{l}\text { Diamètre : } 3.50 \mathrm{~m} \text {; } \\
\text { Longueur : } 820 \mathrm{ml}\end{array}$ \\
\hline Volle de drainage & $\begin{array}{l}29 \text { drains : } \\
\text { Longueur cumulée : } 1300 \mathrm{ml}\end{array}$ \\
\hline
\end{tabular}

gradient de fonte des neiges très important et à l'enlèvement d'éboulis en pied. Une étude géologique, appuyée par des reconnaissances rapides et par la mise en place d'un dispositif d'auscultation pérenne a permis d'émettre différentes hypothèses sur la géométrie du glissement, dont le volume fut estimé entre 500000 et 1 million de $\mathrm{m}^{3}$.

En particulier l'analyse des vecteurs déplacement, reportés sur la figure 4, conduisit à attribuer une forme circulaire à ce glissement (Dubié et al., 1986).

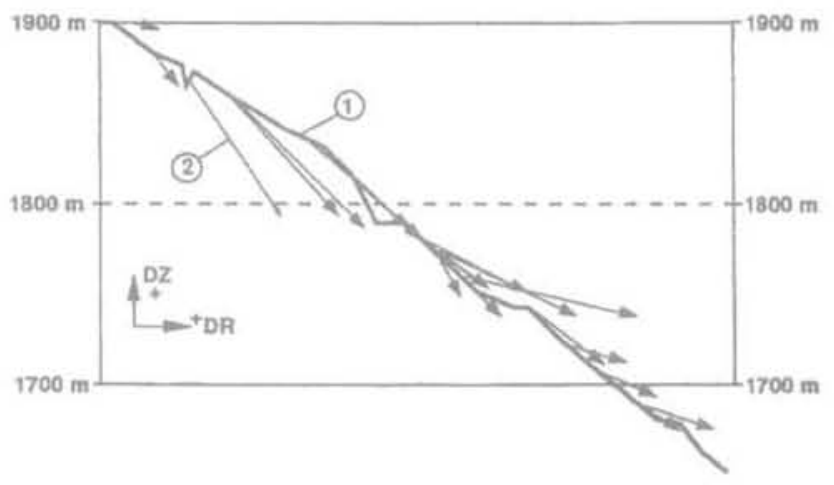

FG.4 Glissement du Billan - Exploitation des mesures géodésiques. 1 : profil du versant; 2: vecteurs déplacements.

Billan slide - Geodesic survey interpretation. 1 ; right bank topography profile ; 2 ; displacements vectors.

Des calculs effectués avec différentes hypothèses géométriques et géomécaniques ont démontré l'efficacité d'un rabattement de nappe au sein du versant (Vibert, 1987). Les résultats de ces calculs sont synthétisés sur la figure 5 . Un mois après la détection du glissement, la décision de forer dans le cristallin une galerie (Fig. 6), complétée par un voile de drainage fut prise. Ce dispositif de drainage permit d'abaisser

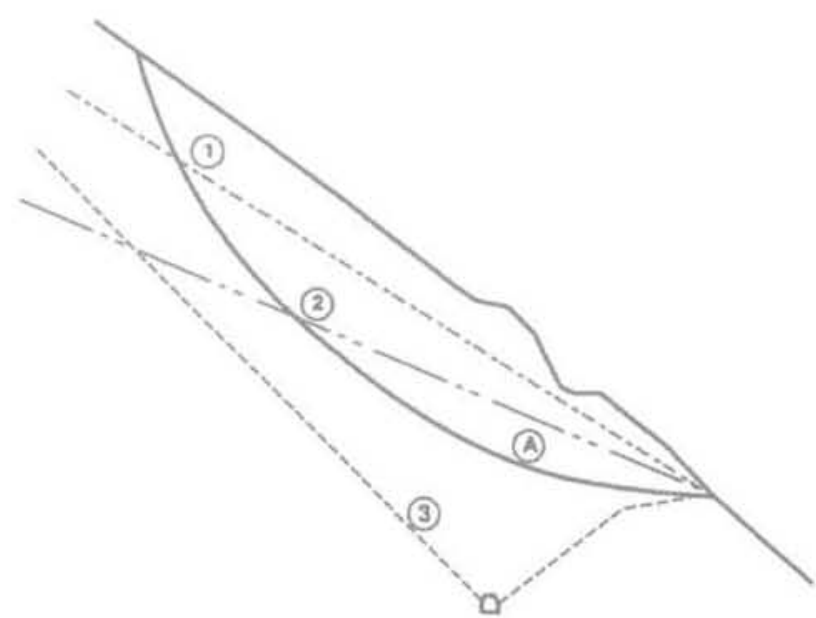

FG.5 Glissement du Billan : influence de la piézométrie sur la stabilité. A : ligne de rupture estimée; coefficients de sécurité suivant la piézométrie : $F_{1}=0,94 ; F_{2}=1,04$; $\mathrm{F}_{\mathrm{3}}=1,16$.

Billan Slide: Influence of piezométric lines on stability. A : estimated failure line: security factors according to different piezometric lines asumptions: $F_{1}=0.94 ; F_{2}=1.04 ; F_{2}=1.16$. 


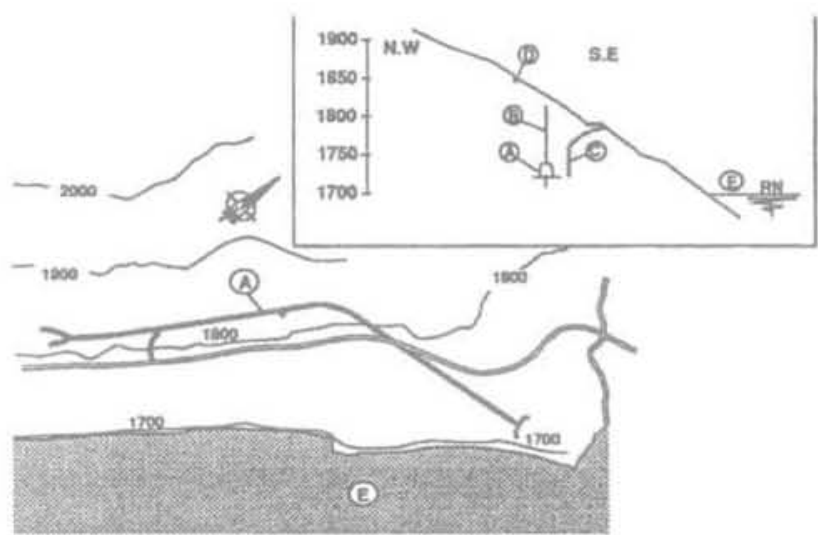

คG.6 Glissement du Billan : dispositif de drainage. $A$ : galeries de drainage $L=1300 \mathrm{~m}$; $\mathrm{B}$ : forages verticaux drainants; C : contacts Lias/Cristallin; D : crevasse de tête du glissement; $\mathrm{E}$ : retenue.

Billan slide : drainage facilities. A : drainage galery boreholes $\mathrm{L}=1,300$; $\mathrm{B}$ : vertical drainage boreholes: C: Lower Jurassic/Cristalline rock contact; D : Landslide uphill crak : $E$; reservoir.

considérablement le niveau piézométrique et de stabiliser le glissement.

Outre le dispositif d'auscultation résumé dans le tableau III, le suivi actuel a été réduit à la surveillance visuelle (avec photos) bi-annuelle de la crevasse de tête et des fissures sur la route.

TABLEAUIII Dispositif d'auscultation pérenne du glissement du Billan.

Billan slide : monitoring system.

\begin{tabular}{l|l}
\hline Nivellement & $\begin{array}{l}51 \text { points de nivellement sur la route } \\
\text { relevés semestriellement }\end{array}$ \\
\hline Photogrammétrie & $\begin{array}{l}\text { Périodicité : quinquennale- } \\
350 \text { cocardes }\end{array}$ \\
\hline Piézométrie & $\begin{array}{l}20 \text { mesures, dont certaines } \\
\text { automatiques et télëtransmises } \\
\text { depuis la galerie de drainage }\end{array}$ \\
\hline Drainage & Mesure automatique en continu \\
\hline Extensométrie & $\begin{array}{l}\text { Chaînes extensométriques à fil invar } \\
\text { en forages }\end{array}$ \\
\hline & $\begin{array}{l}\text { Capteurs de surface au droit } \\
\text { de la crevasse de tête }\end{array}$ \\
\hline
\end{tabular}

Une consigne particulière d'exploitation a été définie dans le cas où la piézométrie du versant et le niveau de la retenue dépasseraient certaines cotes.

L'analyse des données d'auscultation montre que: - des vitesses de déplacements de 5 à $10 \mathrm{~mm} /$ an persistent sur le versant. Elles sont attribuées à la poursuite des phénomènes de fauchage ; ces vitesses sont cohérentes avec la déformation d'ensemble du versant depuis 10000 ans :

- les débits dans la galerie sont très faibles, sauf lors de la fonte des neiges, où ils sont multipliés par 15 (jusqưà atteindre $150 \mathrm{l} / \mathrm{s}$ ) ;

- le niveau piézométrique demeure en dessous de la cote d'alerte.

\section{Carrière de Grand'Maison (Isère)}

L'aménagement de Grand'Maison a nécessité l'extraction de $5300000 \mathrm{~m}^{3}$ de matériaux pour les enrochements, les filtres et les drains du barrage. Les travaux d'extraction ont été réalisés sur 5 saisons durant les mois d'été 1980 à 1984 (Guitton, 1984). Cette carrière, située à $800 \mathrm{~m}$ à l'aval rive gauche de l'actuel barrage, exploitait un versant très raide (pente : $100 \%$ ) constitué de gneiss à schistosité à peu près perpendiculaire au front de taille et à fort pendage (N20 à N25 $\mathrm{E}$ $\left.70^{\circ} \mathrm{E}\right)$.

En juillet 1982, un éboulement de $10000 \mathrm{~m}^{3}$ affecta la partie centrale de la carrière, entre les cotes 1705 et 1740 NGF. Les matériaux provenaient d'un couloir dans lequel affleurait un rocher très fracturé. Ce couloir correspondait à une discontinuité structurale orientée $\mathrm{N} 110^{\circ} \mathrm{E}$ $65^{\circ} \mathrm{N}$, c'est-à-dire oblique au front de taille. Devant le risque de nouveaux éboulements pouvant menacer la stabilité du manteau d'éboulis à forte pente surmontant la carrière dans ce secteur, il fut décidé de limiter l'extraction aux parties amont et aval de cette zone.

En octobre 1982, de nouvelles chutes de blocs d'un volume total de quelques centaines de $\mathrm{m}^{3}$, canalisées par un couloir d'orientation Nord-Sud, affectèrent la partie amont de la carrière. La zone d'alimentation était située $200 \mathrm{~m}$ au-dessus du carreau de la carrière, entre les cotes 1950 et 1980 NGF. Il s'agissait d'une zone de rocher fracturé, altéré et ouvert. Un adoucissement du fruit général de la carrière fut donc entrepris. Parallèlement, une campagne de sismique réfraction et une étude structurale furent décidées.

La sismique réfraction mit en évidence 10 à $25 \mathrm{~m}$ de rocher fracturé et décomprimé (vitesse : $2500 \mathrm{~m} / \mathrm{s}$ sur la figure 7) dans le secteur dominant la carrière.

L'analyse structurale permit de préciser la géométrie de la zone instable. Elle est limitée à la cote 20202030 par des crevasses de tête, repérées lors les études préliminaires. En partie haute, le glissement est limité par une faille d'orientation $\mathrm{N} 100^{\circ} \mathrm{E}$, verticale, alors qu'en partie médiane elle est limitée par les deux réseaux principaux de fracturation $N 70^{\circ} \mathrm{E} 50$ à $90^{\circ} \mathrm{W}$ et $\mathrm{N} 115^{\circ} \mathrm{E}, 55$ à $60^{\circ} \mathrm{E}$.

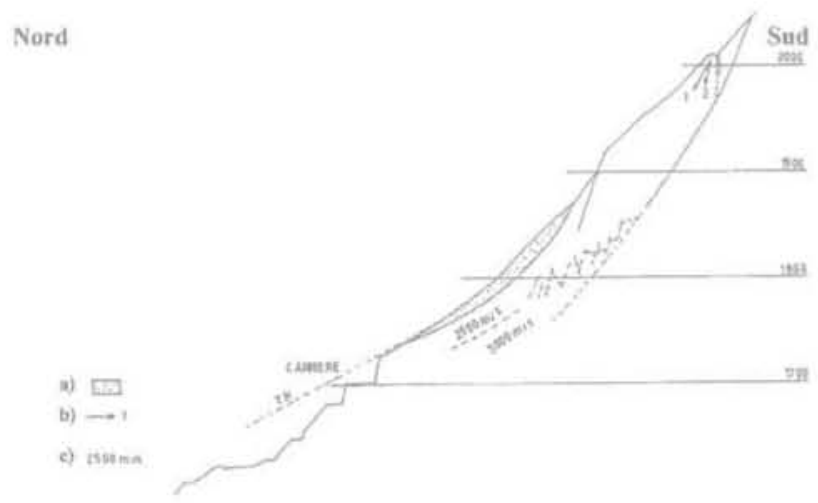

FG.7 Carrière de Grand'Maison : coupe NordSud schématisant la zone instable. a) éboulis ; b) vecteurs déplacements ; c) vitesses sismiques.

Grand'Maison quarry slide ; interpreted section. a) gravity accumulation ; b) displacement vectors; c) sismic velocities. 
Le déplacement des vinchons, mis en place en 1969, montra des déplacements de $5 \mathrm{~cm}$, sans qu'il fût possible de déterminer si ces déplacements étaient le fruit d'une seule grosse crise ou de plusieurs crises successives.

Il fut décidé de déplacer la zone d'exploitation vers l'amont, dans un secteur moins exposé aux chutes de blocs. Compte tenu de l'ampleur de la masse instable, les solutions purge, confortement ou protection passive du chantier durent être abandonnées. En revanche, on procéda à la réduction de la charge unitaire du plan de tir de minage et à la mise en place d'un dispositif d'auscultation pérenne des déplacements des principales fissures ouvertes du versant. Ce dispositif, résumé dans le tableau IV fut soigneusement abrité des chutes de blocs sus-jacents par des structures appropriées (Dubié et Guitton, 1985).

Thalesuir Détail du dispositif d'auscultation de la carrière de Grand'Maison.

Grand'Maison quarry slide: crisis monitoring system.

\begin{tabular}{l|c}
\hline $\begin{array}{l}\text { Capteurs TEDEP (Tẻlécapteurs de } \\
\text { déplacement du CETE de Lyon }\end{array}$ & 10 \\
\hline Cannes de convergence (CETE Lyon) & 9 \\
\hline Cocardes topographiques & 25 \\
\hline Mires à vernier & $\begin{array}{c}\text { Quelques écailles } \\
\text { instrumentées }\end{array}$ \\
\hline
\end{tabular}

L'objectif était de détecter la phase d'accélération des mouvements, annonciatrice d'un éventuel écroulement en masse. Le seuil d'alerte (mise en place de mesures de sécurité sur le chantier) fut fixé à des dépla- cements de $2 \mathrm{~mm} / \mathrm{j}$. Le seuil d'alarme (évacuation du chantier) fut pris égal à 1,5 fois le seuil d'alerte.

Le suivi sur deux saisons des courbes d'ouverture des fissures (Fig. 8) montre de façon flagrante l'influence de la piézométrie liée à la fonte des neiges.

Au total, les déplacements moyens atteignirent $25 \mathrm{~mm}$ en 1983 et $15 \mathrm{~mm}$ en 1984. Remarquons que ces déplacements sur deux ans sont nettement supérieurs aux $50 \mathrm{~mm}$ observés en treize ans sur les vinchons.

\section{3}

\section{La démarche préventive : l'inventaire PPI}

\section{1}

\section{Le cadre réglementaire}

La loi du 22/07/1987 relative à la préparation et à l'organisation des secours en cas de catastrophe comporte des directives concernant les plans d'urgence à mettre en ceuvre à l'aval des barrages.

Ceux-ci, intitulés « plans particuliers d'intervention » (PPI), comprennent une étude de danger.

Le décret du 15/09/1992 introduit une phase préalable à l'établissement du plan d'alerte par le préfet. Dans ce cadre, le maître d'ouvrage du barrage doit remettre au préfet :

- une analyse des risques : notamment séisme et "effondrement de terrain dans la retenue »;

- un projet d'installation des dispositifs techniques de détection et de surveillance.

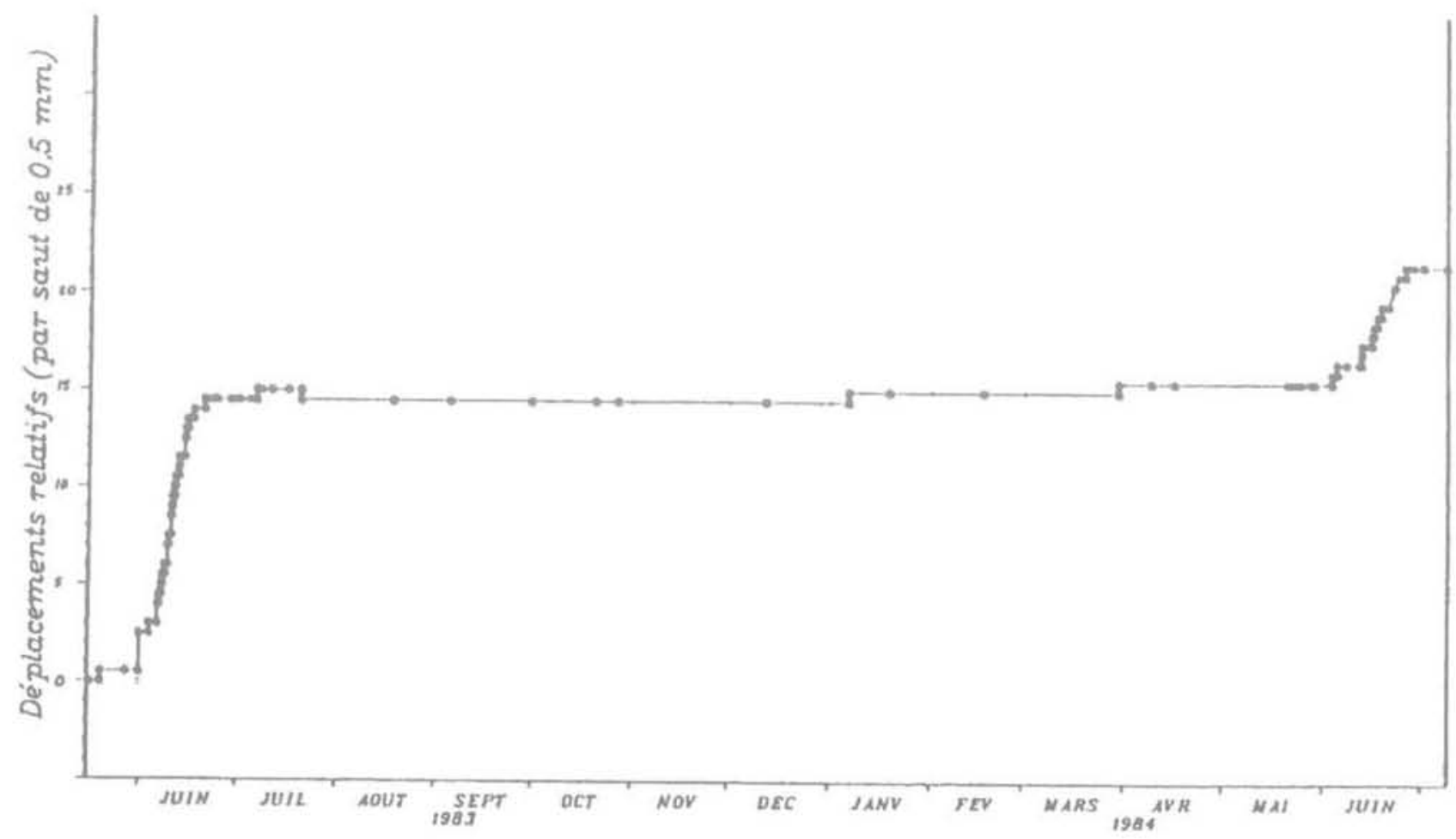

FG. 8 Carrière de Grand'Maison. Capteur TEDEP n 1 : déplacements cumulés mai 1983-juillet 1984. Grand'Maison quarry slide. TEDEP sensor $n^{2} 1$ : total displacements may 1983 -july 1984. 
Enfin, l'arrêté du 01/12/1994 définit plus précisément le contenu de l'analyse des risques. Cette analyse doit comprendre une étude faisant apparaitre le degré d'importance du risque sismique, et « le risque de survenance d'un effondrement de terrain dans la retenue, indépendamment des effets éventuels d'un séisme ».

Sont concernés pour EDF, 67 barrages de hauteur supérieure ou égale à $20 \mathrm{~m}$ et de capacité supérieure ou égale à $15 \mathrm{hm}^{3}$.

\section{2}

\section{La démarche retenue}

L'étude concerne uniquement les effets potentiels d'un mouvement de versant sur un barrage en eau, sans aucune considération pour les éventuels autres effets de ce mouvement (notamment ceux concernant les tiers à l'amont du barrage).

Ces effets sont au nombre de trois :

- création d'une vague ;

- impact direct sur l'ouvrage ;

- risque d'obstruction / partition de la retenue.

La démarche suivie pour cette étude comprend: - une phase de collecte et d'analyse des données existantes, concernant les éventuels mouvements de terrain connus ou potentiels, sur le pourtour de la retenue ou à sa proximité. Ces données sont disponibles soit dans des documents internes à EDF, soit auprès d'organismes extérieurs (RTM, DDE, CETE, SNCF, etc.);

- une phase de terrain consistant à rassembler le maximum d'observations d'ordre géologique, géomorphologique et hydrogéologique, à un instant donné. Certains indices de surface et morphologiques permettent d'identifier des zones susceptibles d'évolution. Les moyens nécessaires à cette phase de terrain peuvent être assez lourds : bateau, hélicoptère, intervention acrobatique en falaise... Cette phase de collecte des données de terrain est guidée et complétée par l'analyse de photographies aériennes;

- une analyse de l'aléa "(mouvement de terrain ») en fonction de la géologie structurale en grand des versants. En effet, une structuration défavorable des formations géologiques dans un versant (plongement des couches dans le sens de la pente, vers la retenue) peut être propice au déclenchement d'un mouvement de terrain en cas de circonstances particulièrement pénalisantes (épisode pluvieux exceptionnel, vidange rapide), tandis qu'une structuration favorable (plongement vers l'intérieur du massif, s'il n'y a pas fauchage) permet d'écarter virtuellement toute potentialité de mouvement de moyenne à grande ampleur.

Chaque zone potentielle de mouvement fait l'objet d'un repérage sur un fond topographique 1/10 000 recensant également tous les indices et observations relatifs à la zone (illustré par la figure 9) et d'une fiche descriptive et analytique autoportante (illustrée par le tableau V).

Les différentes zones de mouvements potentiels ont été classées selon la potentialité d'occurrence d'un glissement dans la retenue suivant une échelle de trois couleurs, définie comme suit :

- potentialité forte (rouge) : la structure et l'état actuel des matériaux sont tels que la modification d'un para-

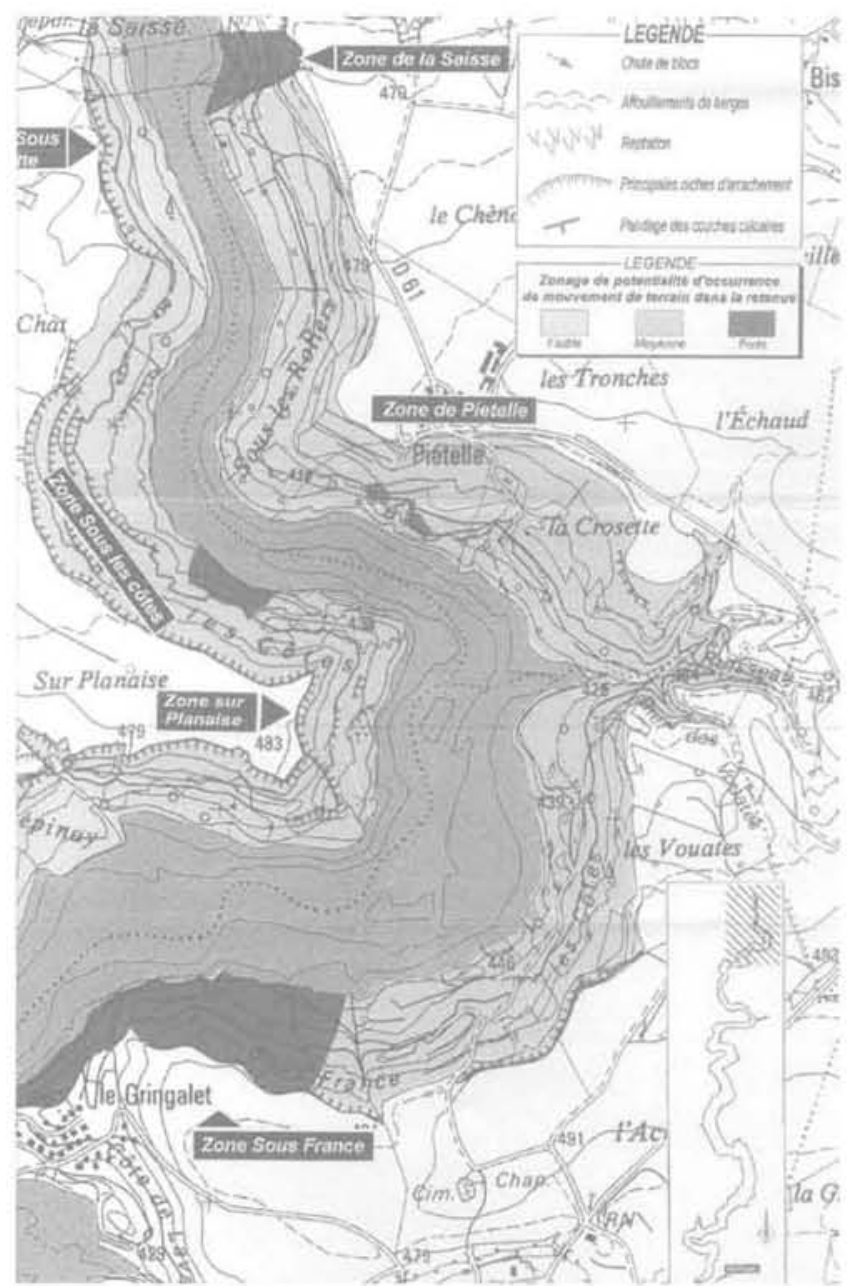

FG.9 Inventaire PPI : carte de potentialité de mouvement de terrain dans la retenue du barrage de Vouglans, partie amont de la retenue.

PPI Inventory: landslides potentiality map in the upstream area of the Vouglans dam reservoir.

mètre (pression interstitielle, cohésion, etc.) peut suffire à la mise en mouvement des masses instables et provoquer leur arrivée dans la retenue ;

- potentialité moyenne (jaune) : une certaine évolution de la zone instable est nécessaire, mais possible, pour se retrouver dans le cas ci-dessus;

- potentialité faible (vert) : ces zones sont reconnues sujettes à des mouvements, ou potentiellement instables, mais:

- soit leur configuration réduit considérablement le risque d'arrivée jusqu'à la retenue (distances importantes),

- soit elle induit un étalement sur le plan spatial (disposition géométrique des matériaux) et/ou temporel (évolution très lente),

- soit elle conduit à l'arrivée dans la retenue de volumes très limités (de l'ordre de quelques centaines de $\mathrm{m}^{3}$ ).

Le rôle de la retenue sur le comportement d'une zone potentiellement instable est signalé, le cas échéant. L'analyse de ce rôle peut parfois conduire à des consignes particulières d'exploitations (vidanges, marnages...). 
TABLEAUY Inventaire PPI : fiche descriptive du glissement du Gringalet sur la retenue du barrage de Vouglans. PPI inventory : landslide descriptive sheet. Example of Gringalet slide in the Vouglans dam reservoir.

\section{Dénomination : Le Gringale}

Mouvement de terrain

\begin{tabular}{|c|c|}
\hline Localisation: & $\begin{array}{l}X: 857,5 \\
\text { Y: } 2178,3 \\
\text { commune : Barésia-sur-Ain } \\
\text { repère : en rive gauche de l'Ain, à } 1200 \mathrm{~m} \text { au Nord-Ouest du village, à proximitẻ des maisons } \\
\text { situées dans l'impasse du lotissement }\end{array}$ \\
\hline Classification du mouvement : & Glissements rotationnels imbriqués \\
\hline Nature des matériaux: & matériaux glacio-lacustres à dominante argileuse claire \\
\hline État actuel d'activité : & Actif \\
\hline Indices d'activitế: & $\begin{array}{l}\text { - niches d'arrachement rẻcentes en tête (cote approximative } 466 \text { NGF, rejet plurimétrique) et } \\
\text { sur l'ensemble du corps du glissement } \\
\text { - nombreuses fissures ouvertes } \\
\text { - arbres basculés et renversés } \\
\text { - morphologie chaotique }\end{array}$ \\
\hline
\end{tabular}

Dimensions:

Versant concerné sur au moins une quarantaine de mètres de hauteur (entre les cotes 466 et 429)

- Largeur : $300 \mathrm{~m}$

Pente moyenne \% :

Supérieure à $30 \%$ dans la partie Sud-Ouest dans la zone encore boisée, entre 25 et $30 \%$ dans la partie centrale du glissement, plus faible $(20 \%)$ dans la partie Nord-Est

Volume:

Difficile à estimer, puisque la partie basse du glissement est immergẻe. Le volume approximatif de la partie émergée est de l'ordre de plusieurs dizaines de milliers de $\mathrm{m}^{3}$

Facteurs déclenchant

Facteurs essentiellement atmosphériques, notamment de fortes périodes pluvieuses, vidange rapide de la retenue

\begin{tabular}{|c|c|}
\hline $\begin{array}{l}\text { Vitesse probable d'arrivée } \\
\text { dans la retenue: }\end{array}$ & Lente (quelques centimètres par heure au maximum) \\
\hline $\begin{array}{l}\text { Potentialité d'occurrence } \\
\text { de glissement: }\end{array}$ & Forte \\
\hline $\begin{array}{l}\text { Potentialité d'occurrence } \\
\text { de glissement dans la retenue: }\end{array}$ & Forte \\
\hline Rôle de la retenue: & $\begin{array}{l}\text { Pied du glissement baigné par la retenue : risque de mise en charge plézométrique lors d’une } \\
\text { vidange rapide de la retenue }\end{array}$ \\
\hline \multicolumn{2}{|l|}{ Risque } \\
\hline Nature du risque: & $\begin{array}{l}\text {-Alluvionnement de la retenue } \\
\text {-Obstruction? }\end{array}$ \\
\hline Ampleur du risque : & Faible, vis-à-vis de la dimension probable de l'éventuel bouchon \\
\hline \multicolumn{2}{|l|}{ Remarques complémentaires } \\
\hline \multicolumn{2}{|c|}{ 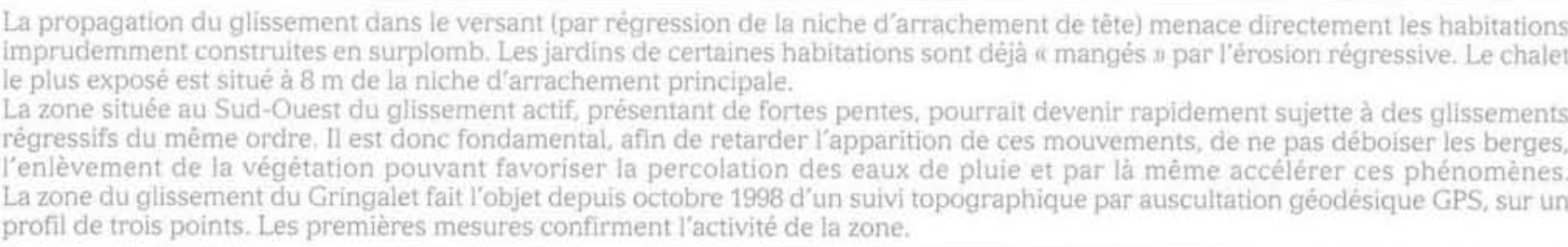 } \\
\hline
\end{tabular}

\section{3}

\section{Les résultats}

Au total, 255 mouvements de terrain déclarés ou potentiels ont été recensés sur les versants des 67 retenues étudiées (Thomaïdis, 2000). Cinquante-huit pourcent de ces mouvements ont été classés en vert (potentialité d'occurrence dans la retenue faible) et $23 \%$ en rouge (potentialité d'occurrence forte). Parmi ces 255 mouvements déclarés ou potentiels, 65 sont suivis : 31 sont surveillés par l'exploitant (surveillance visuelle, prise régulière de photos...) et 34 sont auscultés.

La répartition de ces 34 zones auscultées montre que : 


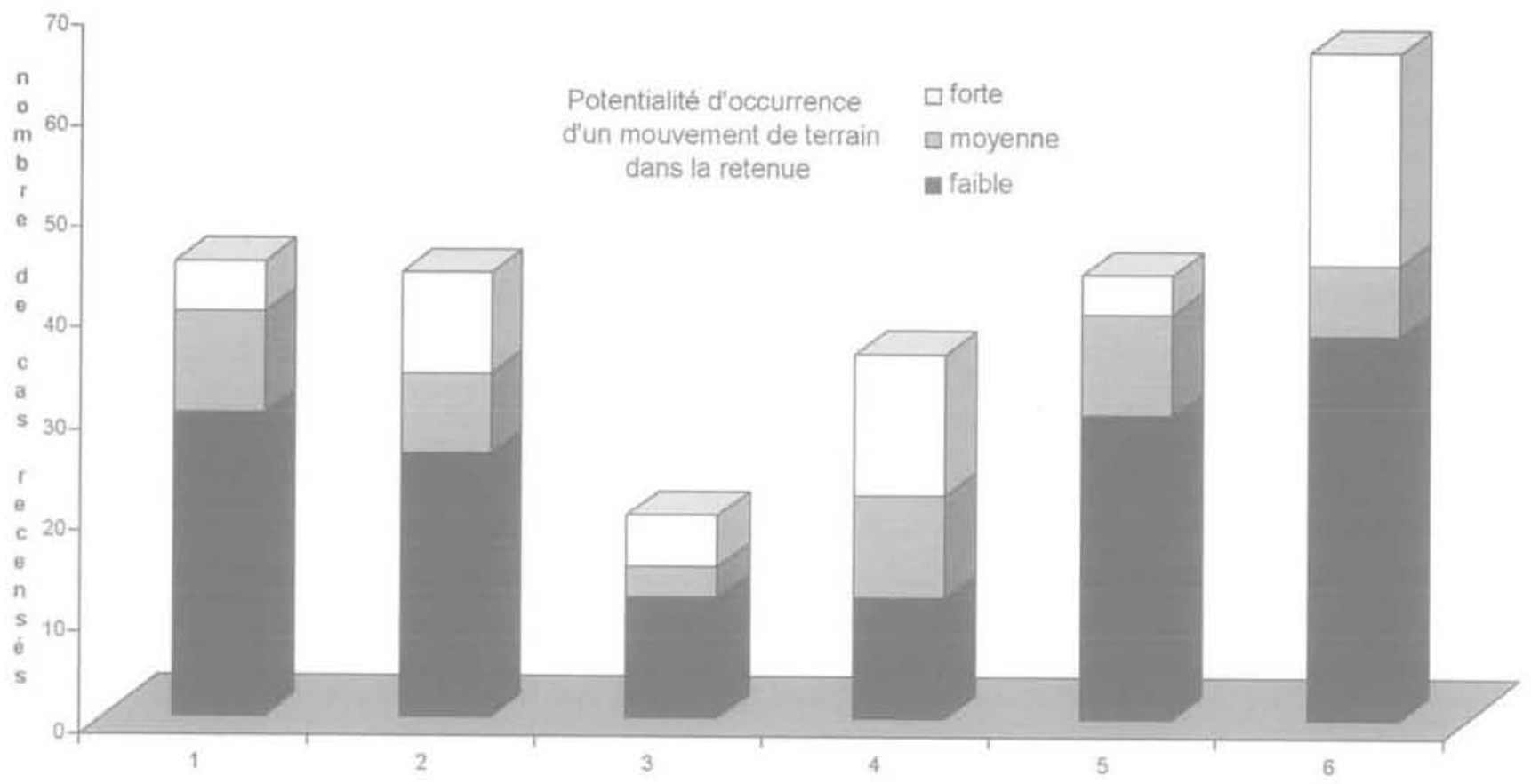

FG. 10 Répartition des 255 mouvements de terrain déclarés ou potentiels recensés sur les 67 retenues EDF. 1) chutes de blocs ; 2) éboulement par basculement ou cisaillement en pied ; 3) éboulement et glissement banc sur banc ; 4) glissements rotationnels; 5) glissement de matériaux initialement cohérents ayant perdu leur cohésion suite à des phénomènes de versant ; 6) écoulements (fluage, solifluxion, ravinement et coulées boueuses).

Distribution of the 255 known or potential slope movements affecting the 67 biggest reservoirs. 1) rockfalls ; 2) tilting or rough translationnal slides ; 3 ) simple translationnal slides; 4) circular slides : 5) slides in materials which lost their cohesion in side mountain events (weathering, down-bending) ; 6) flows (creepings, viscous and muddy flows; gully erosion).

- 19 zones (soit $56 \%$ des cas) sont concernées par l'aléa création d'une vague ;

- 9 zones (soit $27 \%$ des cas) sont concernées par l'aléa partition/obstruction :

- 4 zones soit $12 \%$ sont concernées par l'aléa impact direct sur l'ouvrage ;

-2 zones sont concernées par des aléas qui ne rentrent pas dans le cadre des PPI (stabilité de pylônes, affaissements routiers).

La figure 10 illustre la répartition de ces 255 mouvements de terrain en fonction du type de mouvement mis en jeu et de la potentialité d'occurrence associée.

Les chutes de blocs caractérisent des éboulements de masses rocheuses éparses et/ou étalées dans le temps. Le risque associé est un impact direct sur l'ouvrage, ce qui représente une faible proportion des chutes de blocs recensées. C'est par exemple le cas au barrage de Pla de Soulcem, qui a nécessité des dispositions particulières de protection et d'alarme.

Le risque de création de vague pouvant submerger le barrage est associé aux mouvements pouvant mettre en jeu, à de fortes vitesses, des volumes très importants. Le risque concerne essentiellement les éboulements de type banc sur banc (cas de la Pelloud) et les éboulements par basculement/cisaillement en pied (cas du Chastel), pour lesquels la cinétique de glissement est brutale et imprévisible. Dans une moindre mesure, il concerne aussi les glissements rotationnels (cas du Billan, suite à des phénomènes de versant ayant conduit à une perte de la cohésion en grand du massif). Sur ces trois types de glissement ( $56 \%$ des cas recensés), $23 \%$ ont une potentialité forte, La création de la vague est fonction de la vitesse d'arrivée, mais aussi de la surface du front de glissement dans la retenue. Afin de quantifier le risque induit pour l'ouvrage, des modélisations prenant notamment en compte la géométrie de la retenue sont nécessaires.

Le risque d'obstruction/partition existe pour tous les types de glissements, mais en particulier pour les glissements rotationnels dans les matériaux meubles et pour les écoulements (coulées de boues, solifluxion...). En général, les vitesses et les volumes élémentaires demeurent trop faibles pour présenter un risque réel pour l'ouvrage. L'impact des mouvements de versant se traduit alors par un alluvionnement et une perte de la capacité utile du réservoir. C'est par exemple le cas des glissements en loupe de la queue de la retenue de Vouglans.

\section{4}

\section{Risque d'impact direct sur le barrage : appui rive gauche du barrage de Pla de Soulcem (Ariège)}

Le barrage de Pla de Soulcem est un barrage en terre à masque amont de $66,5 \mathrm{~m}$ de hauteur, implanté sur un verrou glaciaire d'altitude (1600 NGF).

Le versant rive gauche, très raide $(100 \%)$ est constitué de gneiss micaschisteux, injectés de nombreuses veines de granite pegmatitique. Ce rocher, montrant de nombreux polis glaciaires, est découpé par deux failles F1 et F2, inclinées de $45^{\circ}$ vers la vallée, visibles sur la figure 11. Ces deux failles parallèles, distantes d'une trentaine de mètres, délimitent un parallélépipède d'un volume de $260000 \mathrm{~m}^{3}$ dominant directement l'ouvrage (Vaast, 1997). 


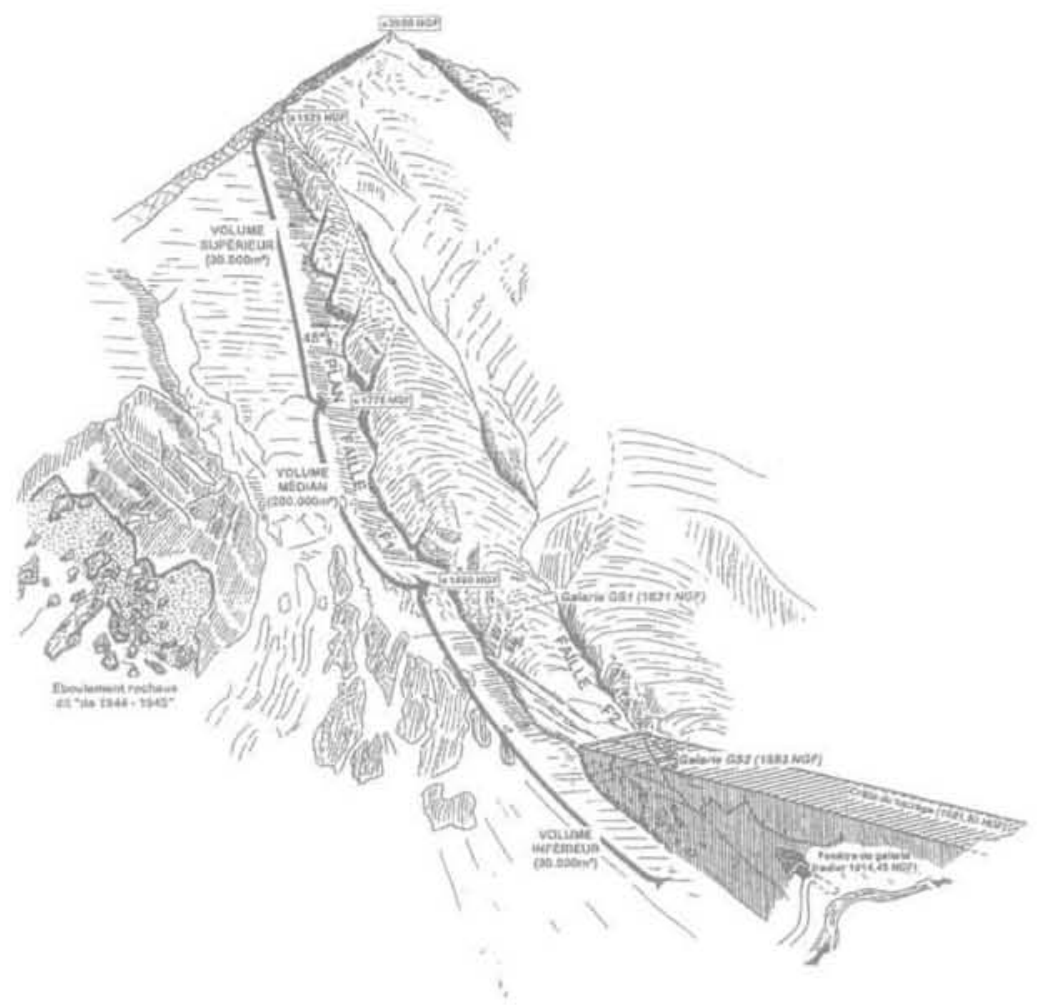

FIG. 11 Barrage de Pla de Soulcem: croquis géologique de l'appui rive gauche.

Pla de Soulcem dam: geological drawing of the left abutment showing potential rockfalls areas.

Le risque d'un éboulement massif de l'ensemble du parallélépipède fut pris en compte dès le projet. Deux galeries de reconnaissances démontrèrent que les deux failles, ouvertes de près d'un mètre en surface, se refermaient rapidement en profondeur. La faille F1, la plus à l'amont, est marquée par la présence de mylonite argileuse d'une épaisseur de $2 \mathrm{~m}$ par endroits. L'ouverture en surface de ces discontinuités structurales fut attribuée à la poussée amont-aval du glacier, tendant à décoller le parallélépipède du reste du massif.

L'hypothèse d'un écroulement brutal fut donc écartée car le parallélépipède s'enracine dans le versant et en pied.

Quatre pendules inversés furent toutefois mis en place dans les deux galeries. Ils forment le maillon essentiel du système de prévention d'un éboulement en masse, qui serait précédé de lentes déformations du versant. Depuis 19 ans, aucun déplacement n'est observé.

En revanche, l'arête amont du parallélépipède, très découpée et très décomprimée, laisse craindre des chutes de blocs pouvant endommager l'ouvrage. Le parement amont du barrage a donc été dimensionné pour résister à des impacts de volumes rocheux de 100 à $150 \mathrm{~m}^{3}$ arrivant avec des vitesses de $100 \mathrm{~km} / \mathrm{h}$. Concrètement, le masque d'étanchéité amont est doublé par une couche ( semi-imperméable » d'une épaisseur minimale de $8 \mathrm{~m}$, capable d'assurer temporairement une étanchéité suffisante en cas de perforation du masque bitumineux pour ne pas mettre en péril la stabilité de l'ouvrage.

Un dispositif d'alerte est composé de plusieurs géophones disposés sous le parement du barrage, télétransmis à l'usine. Ces géophones enregistreraient les 174 vibrations liées à des impacts de gros volumes sur le parement. A ce jour, aucune chute de bloc n'a été relevée.

\section{2}

Risque de création d'une vague : l'éperon de la Pelloud (retenue du barrage de Monteynard, Isère)

Le barrage de Monteynard est un barrage-voûte épais de $135 \mathrm{~m}$ de hauteur, barrant la vallée du Drac et créant une retenue de $275 \mathrm{hm}^{3}$.

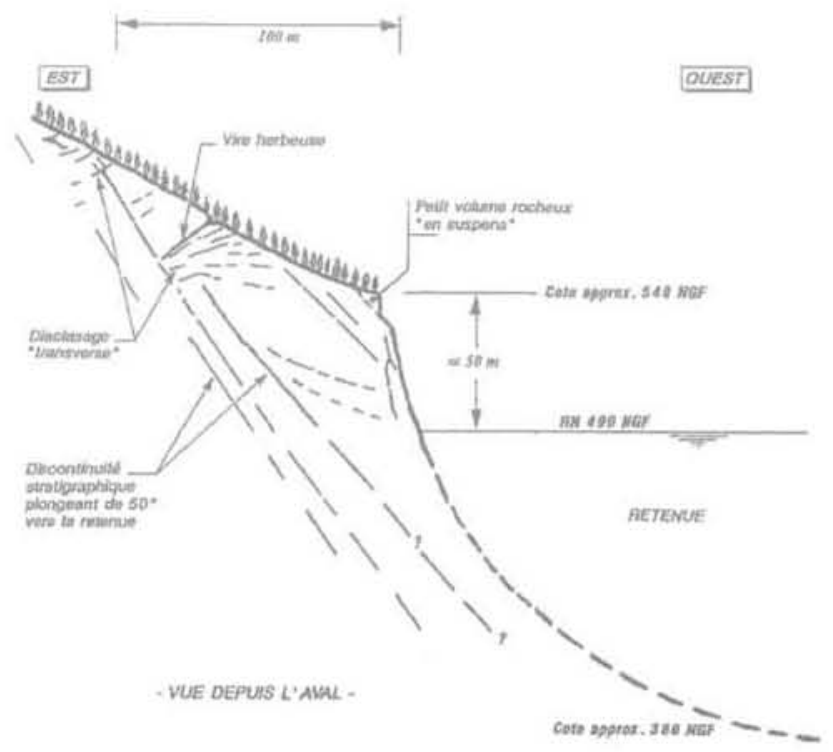

FIG. 12 Coupe du promontoire de la Pelloud. Section through la Pelloud promontory. 
Le promontoire rocheux de la Pelloud est situé à $1500 \mathrm{~m}$ à l'amont rive droite du barrage. Il est formé de calcaires marneux du Lias fracturés et légèrement métamorphisés.

Les plans de stratification, pentés de 45 à $50^{\circ}$ vers la retenue, laissaient craindre le glissement banc sur banc d'un volume monolithique de plusieurs centaines de milliers de $\mathrm{m}^{3}$, non buté en pied, comme l'illustre la figure 12.

Le système d'auscultation anciennement mis en place, qui consistait en un réseau d'observation angulaire avec des mesures de périodicité annuelle et un réseau de nivellement avec des mesures de périodicité quinquennale, se révéla inadapté à l'aléa.

Une reconnaissance en falaise de la nature et de l'état de la stratification fut décidée. Elle révéla que les hypothétiques plans de glissement étaient des joints fermés, recoupés par la schistosité verticale et disparaissant localement entre les plans de schistosité et de fractures. Ces joints, très ténus, sont pratiquement invisibles en falaise, et ne demeuraient visibles que depuis la rive opposée, car ills sont soulignés par un léger sous-cavage de gélifraction (Vaast et al,, 1998), Le risque de glissement banc sur banc fut par conséquent écarté, car les plans de glissement, ondulés et très rugueux en grand, présentent un angle de frottement et une cohésion suffisante.

L'intervention en falaise, délicate à mettre en ceuvre, se révéla donc très efficace puisqu'elle permit d'éviter des investigations lourdes et coûteuses.

\section{$3: 33$}

\section{Risque d'obstruction/partition : les glissements de la queue de la retenue du barrage de Vouglans (Ain)}

Le barrage de Vouglans est un barrage voûte de $103 \mathrm{~m}$ de hauteur, mis en service en 1970. Il forme une retenue de $605 \mathrm{hm}^{3}$ sur la rivière Ain.

La partie amont de la retenue se développe au sein de formations glacio-lacustres quaternaires à dominante argileuse. L'Ain entaille et déstabilise ces forma- tions sur près de $50 \mathrm{~m}$ de hauteur. La totalité des berges de cette partie de la retenue a été ou est le siège de puissants glissements en loupe. De vastes amphithéâtres, parfois doubles ou triples, traduisent des glissements emboités. Lors de la crise de 1970 (Fig. 13), $1300 \mathrm{~m}$ de berge furent affectés entre les cotes 429 et 466 , c'est-à-dire sur toute la hauteur du versant : zone "sous les cotes » sur la figure 9 .

Les essais en laboratoire montrent que ces matériaux glacio-lacustres sont très sensibles au remaniement et à la variation de leur teneur en eau (Devèze, 1999). En particulier, la consistance des matériaux varie brutalement pour d'infimes variations de leur teneur en eau. On distingue donc au sein des amphithéâtres la partie supérieure, où les glissements sont des glissements rotationnels typiques, de la partie inférieure où les matériaux atteignent la retenue sous forme de coulées (le remaniement des matériaux est alors maximal).

L'action de la pluje est hautement défavorable à la stabilité des glissements, en diminuant les caractéristiques géotechniques des matériaux. En revanche, il est établi que la mise en eau du barrage n'a pas eu d'effet déclencheur sur d'éventuels glissements. Elle a diminué la sape de pied auparavant exercée par la rivière et diminué les gradients hydrauliques de la nappe dans le versant. Cependant, des vidanges rapides pourraient mettre en péril certaines berges instables. La consigne d'exploitation est donc adaptée à ce risque, en minimisant les marnages de la retenue.

Les principaux glissements actifs sont auscultés au moyen de 13 profils géodésiques mesurés par satellite (GPS), comportant au total 56 repères mesurés annuellement. Les vitesses maximales dépassent $10 \mathrm{~cm} / \mathrm{an}$ dans les zones de tête des glissements les plus actifs. L'aléa concerne donc la partition de la retenue dans sa partie la plus étroite. Un profil bathymétrique quinquennal est réalisé dans une zone étroite où deux glissements actifs se font face.

Un risque existe également pour les habitations imprudemment construites à proximité des berges. La régression de la zone d'arrachement peut à terme menacer les constructions.

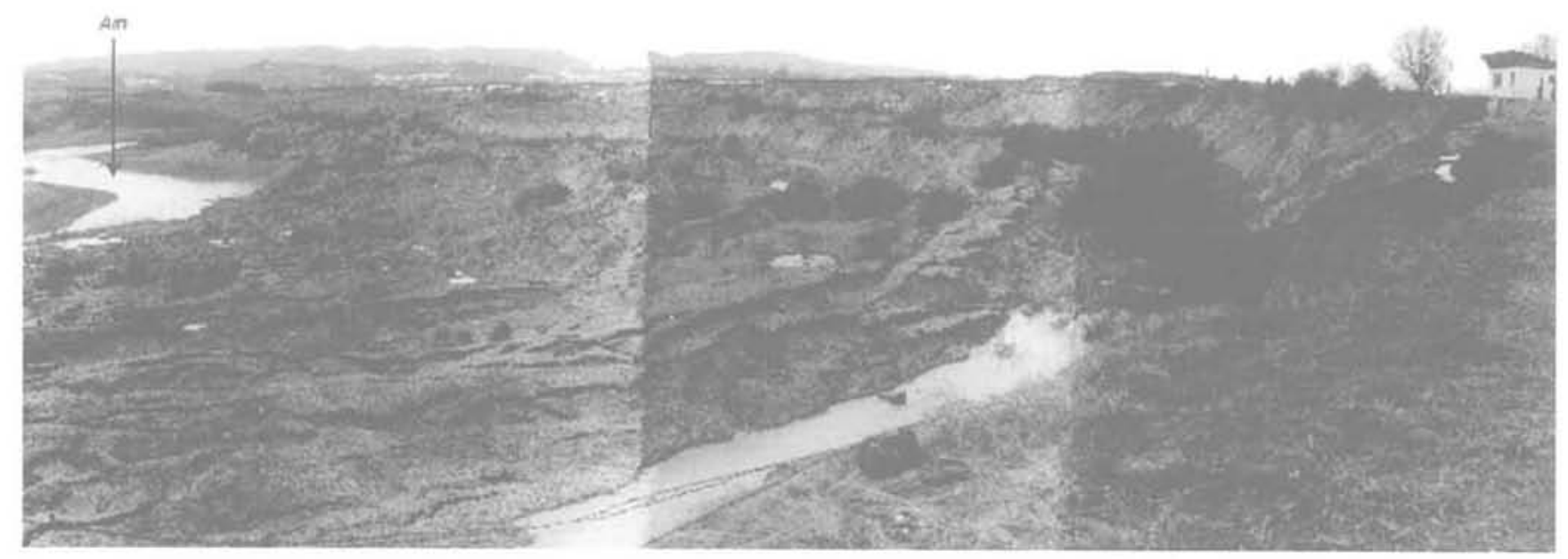

FG.13 Le glissement « sous les Cotes » après la crise de 1970. "Sous les Cotes n slide in 1970. 


\section{Gestion du risque à Électricité de France}

L'expérience d'Électricité de France dans la gestion des mouvements de versants sur les retenues de barrage met en évidence deux points fondamentaux qui peuvent impacter directement la sûreté des installations hydroélectriques :

1) La rapidité de réaction en cas de crise est primordiale. Cette réaction peut se résumer à deux actions: - mise en place immédiate d'un dispositif d'auscultation sommaire et rustique (vinchons sur fissures, repères sur chaussée...) et suivi attentif de diverses données naturelles (débits des sources, apparition de nouvelles sources ou de nouvelles fissures). Ils permettent d'acquérir les paramètres de crise (déplacement total, vitesses maximales), qui se révèlent par la suite souvent fondamentaux dans la quantification du risque :

- diagnostic géologique sommaire, qualifiant le glissement en terme d'aléa. Ce diagnostic rapide peut conduire à limiter le cas échéant l'indisponibilité liée à la baisse préventive du plan d'eau ou peut conduire à des actions préventives lourdes (évacuation...).

2) Un diagnostic géologique approfondi est indispensable. S'appuyant sur un travail de bureau (dépouillement d'archives concernant la zone instable, comparaison de photos aériennes), de données de terrain (géologie structurale, lithologie, piézométrie...) et souvent complété par des reconnaissances adaptées (sondages, piézomètres), il permet de quantifier le risque et de dimensionner au plus juste un dispositif pérenne d'auscultation et de suivi. suite :

Ces deux points fondamentaux permettent par la - de préciser les consignes d'exploitation du barrage et du plan d'eau;

- de définir les données d'entrée, en termes de géométrie, vitesses, piézométrie, caractéristiques géomécaniques, d'un éventuel modèle de calcul;

- de préciser les seuils d'alerte :

- d'adapter et d'optimiser un éventuel traitement.

Les actions proposées par EDF dans la gestion des mouvements de versants sur les grands ouvrages hydroélectriques sont systématiquement soumises à l'approbation du Comité technique permanent des grands barrages (CTPB).

L'exploitant du barrage est le maillon essentiel du dispositif de gestion du risque. Il recueille les données du dispositif d'auscultation, assure la surveillance visuelle des versants de sa retenue et déclenche le cas échéant une procédure d'alerte. Sa sensibilité aux phénomènes de mouvements de versant est donc fondamentale. Il doit être en mesure d'analyser sommairement les données qu'il acquiert et doit être attentif aux indices d'activation (ou de réactivation) d'un glissement de terrain. Des stages de sensibilisation, animés par des géologues, sont régulièrement mis en place dans cette optique.

\section{Bibliographie}

Bordet C. et un groupe de Travail du Comité français des grands barrages - « Études et travaux réalisés en France en raison de I'Instabilité de versants de retenues $1,14^{*}$ congrès de la Commission internationale des grands barrages, Rio de Janeiro, 1982, p. 563-589.

Bozonnet J.P. - " Aménagement de Puylaurent. Glissement du Chastel v. Note qéologique $n^{\prime 3} 3$. Synthèse des reconnaissances réalisées sur le site au 01/09/1996, Document interne EDF.

Chowdhury R. - $\alpha$ Analysis of the Vajont Slide. New approach $x_{.}$ Rocks Mechanics 11, 1978, p. 29-38.

Devéze G. - " PPI Retenue de Vouglans. Étude de la stabilité des rives n. Document interne EDF, 1999.

Dubié J.Y. - " Aménagement de Grand'Maison. Versant rive droite de la retenue supérieure. Reconnaissances et auscultation $x$. Document interne EDF, 1987.

Dubié J.Y.. Bénéfice P., Blais J.P. - * Aménagement de Grand'Maison. Glissement du Billan ». Dossier de présentation au Comité technique permanent des grands barrages, 1986.

Dubié J.Y., Bénéfice P., Guitton C. - « Télétransmission de l'auscultation d'un glissement : retenue de Grand'Maison, glisse-

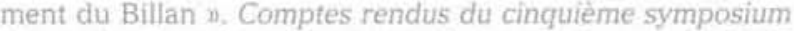
international sur les glissements de terrain, Lausanne, 10-15 juillet, 1988.

Dubié J,Y., Guitton C. - « Carrière du barrage de Grand'Maison. Auscultation du versant ». Réunion Travaux de qénie civil et laboratoires, Aix-en-Provence, 1985, Direction Équipement d'EDF, p. 1-12.

Duffaut P., Dubié J.Y. - « Management of steep rock slopes after studies on reservoir slopes by Élecricité de France 1,8 th Int. Symp. On Landslides, Cardiff, 2000.

Guitton C - « Carrière du barrage de Grand'Maison : données du projet, modalités d'exploitation, dispositif d'auscultation 1. Industrie Minérale - Les Techniques 1984, p. 771-785.

Thomaìdis C. - « Note de Synthèse de retour d'expérience sur les 67 PPI ». Document interne EDF, 2000.

Vaast J.S. - « PPI retenue de Soulcem. Étude de la stabilité des rives n. Document interne EDF, 1997.

Vaast J.S., Durouchoux C., Devèze G. - « PPI Retenue de Montey. nard. Etude de la stabilité des rives 11. Document interne EDF, 1998.

Vibert C. - « Programmes de stabilité de versants ou de berges de réservoir. Application au glissement du Billan (aménagement de Grand'Maison) 1. Document interne EDF, 1987. 